\title{
Anomalous and planar Nernst effects in thin films of the half-metallic ferromagnet $\mathrm{La}_{2 / 3} \mathrm{Sr}_{1 / 3} \mathrm{MnO}_{3}$
}

\author{
Cong Tinh Bui and F. Rivadulla* \\ Centro de Investigación en Química Biológica y Materiales Moleculares (CIQUS), Universidad de Santiago de Compostela, \\ 15782 Santiago de Compostela, Spain
}

(Received 2 June 2014; revised manuscript received 6 August 2014; published 4 September 2014)

\begin{abstract}
We report the planar and anomalous Nernst effects in epitaxial thin films of spin polarized $\mathrm{La}_{2 / 3} \mathrm{Sr}_{1 / 3} \mathrm{MnO}_{3}$. The thermal counterpart of the anomalous Hall effect in this material (i.e., the anomalous Nernst effect) shows an extreme sensitivity to any parasitic thermal gradient, resulting in large asymmetric voltages under small temperature differences. This should be considered when interpreting the magnitude of the electrical response in nanostructures and devices that operate under high current densities. Finally, none of the observed magnetothermoelectric signals is related to the spin Seebeck effect in this material.
\end{abstract}

DOI: 10.1103/PhysRevB.90.100403

PACS number(s): 73.50.Lw, 85.80.Lp

The discovery of the intrinsic spin Seebeck effect (SSE) in magnetic materials, irrespective of their conductivity, opens unforeseen possibilities for the creation and manipulation of pure spin currents (spin caloritronics) as well as of energy harvesting [1-3]. Basically, when a thermal gradient is established parallel to the magnetization of a ferromagnet (FM), spin angular momentum is transported along the system in response to the temperature difference. This time-varying magnetization is able to pump a pure spin current at the interface with a paramagnetic metal (normally Pt), which is then transformed into a transverse electrical current via the inverse spin Hall effect [4].

In FM metals, the different densities of states and Fermi velocities for the spin up and spin down populations produce different conductivities for the opposite spin directions [5]. Therefore when the spin lifetime is larger than the momentum relaxation time, a spin-dependent Seebeck and Peltier coefficient is predicted, on the basis of the Onsager reciprocities [6].

However, the observation of a clear SSE signal in magnetic insulators demonstrates that its origin must be different from the conventional Seebeck effect in magnetic conductors, though their phenomenology is very similar [2]. Although the role of spin-phonon coupling through the substrate was emphasized to explain its long-range nature [7,8], the microscopic mechanism is still under debate. On the other hand, recent studies on metallic FM permalloy have shown that the anomalous Nernst effect (ANE) may be an important contribution to the measured SSE in some materials [9-11].

Therefore, it is of fundamental interest to explore the nature and magnitude of spin-dependent thermoelectric effects in novel technological materials, and thereby provide a better understanding of the delicate balance between spin, charge, and heat currents in nanodevices [12-16].

In this Rapid Communication we report the observation of an intrinsic planar Nernst effect (PNE) and anomalous Nernst effect (ANE) in thin films of $\mathrm{La}_{2 / 3} \mathrm{Sr}_{1 / 3} \mathrm{MnO}_{3}$ (LSMO). This material shows a fully spin polarized $3 d$ band [17], and a $T_{C} \approx 360 \mathrm{~K}$, which motivated its extended use as a FM electrode in tunnel junctions [18]. We show that through a very careful control of the thermal gradients in the thin films, the ANE can

*f.rivadulla@usc.es be completely suppressed and separated from the symmetric PNE response. We further demonstrate that there is a perfect correspondence between the magnetothermal effects and their electrical counterparts in LSMO. Our findings also establish an upper limit for the possible observation of SSE in this system. These findings are relevant for a better understanding of the spin-dependent thermoelectric phenomena in similar correlated metallic oxides.

Before discussing the results, we briefly recall the physical quantities that govern the magnetothermoelectric effects in our system. In magnetic conductors, the spin-orbit interaction introduces an anisotropic thermoelectric voltage depending on the angle $\Theta$ between the temperature gradient and the magnetization $M$ [6]. These are the thermal counterparts (Onsager reciprocals) of the anisotropic magnetoresistance (AMR) and planar Hall effect (PHE) [19]. For the PNE, the transverse voltage $V_{x y}$ is related to $M$ and $\Theta$ by $[10,20]$

$$
S_{x y}=\frac{V_{x y}}{\nabla T_{x}} \propto|M|^{2} \sin \Theta \cos \Theta,
$$

with $M$ and $\nabla T$ both lying in the $x y$ plane.

However, in a conducting FM, any $\Delta T_{z} \neq 0$ will create a measurable $V_{x y}$ response due to the anomalous Nernst effect (ANE) [11],

$$
\nabla V_{x y}=-S_{x x} \xi\left(\hat{m} \times \nabla T_{z}\right),
$$

where $S_{x x}$ is the linear Seebeck coefficient, $\hat{m}$ is the unit vector of the magnetization, and $\xi$ is the Nernst factor [12].

The experimental setup for the measurement of the magnetothermoelectric effects is shown in Fig. 1 (see the Supplemental Material for details [21]). We have used the same transverse configuration as normally used to measure the SSE. A small $\Delta T_{x} \leqslant 0.8 \mathrm{~K} / \mathrm{mm}$ was always used in order to be within the linear, reversible regime for the thermopower, and to avoid any uncontrolled temperature gradient in the other direction. In addition, we have also determined the transverse voltage for a cross-plane thermal gradient $\left(\Delta T_{z} \neq 0\right)$ while keeping the in-plane temperature constant $\left(\Delta T_{x}=0\right)$.

The in-plane angular dependence of the magnetization of LSMO is show in Fig. 1(c). The result is the characteristic of a system with biaxial symmetry, with the easy axis along the [100] direction of the film [22]. The transverse voltage $V_{x y}$ was recorded as a function of the magnetic field for different angles $\Theta$ between $\Delta T_{x}$ and $M$ [according to the easy axis direction 

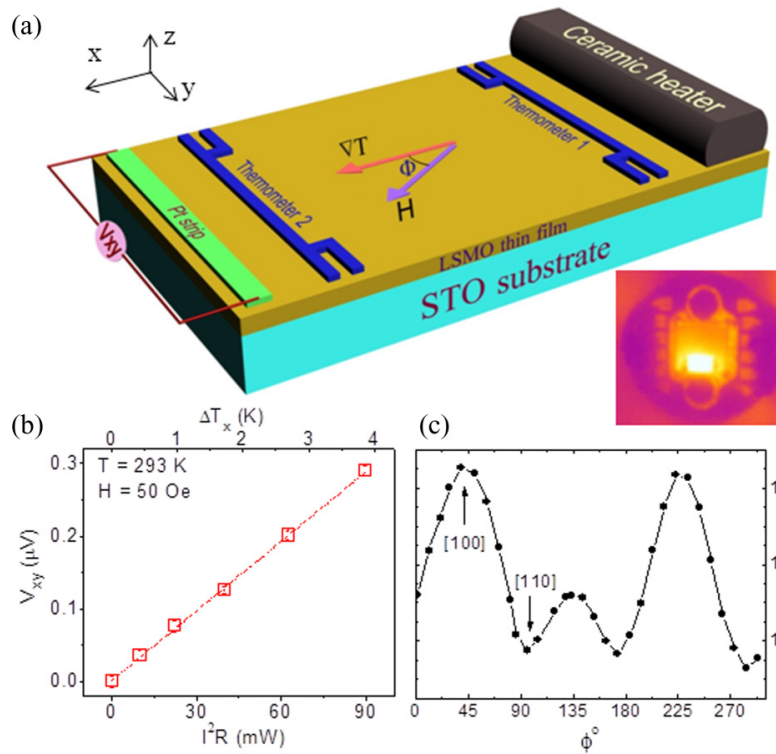

(c)

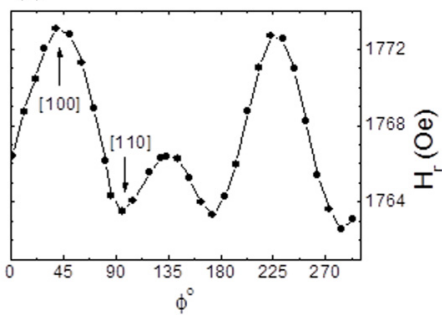

FIG. 1. (Color online) (a) Sketch of the device used to measure the PNE and ANE in a thin film (35 nm thick) of LSMO, along with a thermal image of the actual device with $\Delta T_{x} \neq 0$. The two thermometers are Pt resistances deposited by optical lithography (see the Supplemental Material [21]). (b) Evolution of the transverse voltage with the heating power and $\nabla T_{x}$. (c) Angular dependence of the resonance field of the ferromagnetic resonance line $(9.4 \mathrm{GHz})$, showing the crystalline directions of the easy and hard axis in LSMO. Based on these measurements, the angle between the $M$ and $\nabla T_{x}$ in (a) is $135^{\circ}$.

shown in Fig. 1(c)]. The results are summarized in Fig. 2: The absolute value of $V_{x y}$ increases with the applied magnetic field, until saturation is reached at $H_{\text {sat }}(\sim 43$ Oe). The amplitude of $V_{x y}$ at saturation depends linearly on the temperature gradient [Fig. 1(b)]. Furthermore, all curves collapse when divided by the corresponding temperature gradient (see the Supplemental Material, Fig. S3 [21]), indicative of its thermoelectric origin, and demonstrates the accurate determination of $\nabla T_{x}$ by the two $\mathrm{Pt}$ resistances. Finally, the appearance of symmetric behavior in all curves indicates that the PNE is certainly the driving mechanism of the observed effect [10].

The amplitudes of the magnetothermoelectric power $S_{x y}$ and $H_{\text {sat }}$ both drop to zero when the base temperature approaches $T_{\mathrm{C}}$ [Fig. 2(b)], demonstrating their intimate relationship to the spontaneous magnetization.

The results of $V_{x y}$ at $293 \mathrm{~K}$ (with $\Delta T_{x}=3.9 \mathrm{~K}$ ) when rotating the magnetic field are show in Fig. 2(c), and the normalized magnetothermopower $S_{x y} / \Delta S$ is shown in Fig. 2(d), for different base temperatures. The angular dependence of $S_{x y}$ shows a good agreement with the predictions of Eq. (1) for PNE, at all the temperatures probed in this work [Fig. 2(d)].

The PNE has its origin in the spin-orbit interaction, and should therefore present a perfect correspondence with the PHE in the same material [20]. In order to verify such a correspondence in the LSMO, we have measured the AMR $\left(R_{x x}\right)$ and PHE $\left(R_{x y}\right)$ in thin films grown under the same conditions as those described above. The results are shown in Fig. 3. Both AMR and PHE are observed in LSMO at room temperature $\left(T \ll T_{C}\right)$ [Figs. 3(a) and 3(b), respectively].
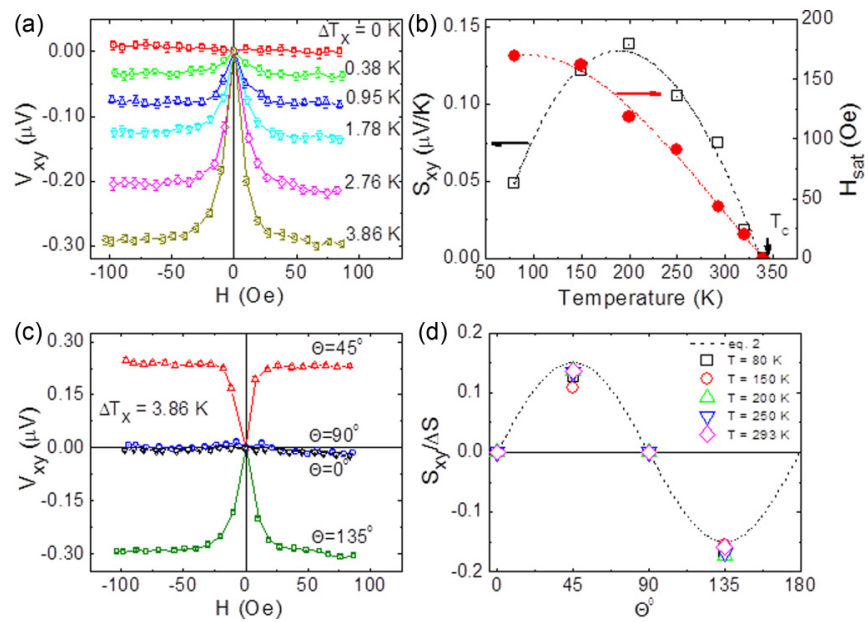

FIG. 2. (Color online) (a) Field dependence of the transverse voltage $V_{x y}$ at $293 \mathrm{~K}$ for different temperature gradients $\nabla T_{x}$ at $\Theta=$ $135^{\circ}$. (b) Temperature dependence of the transverse thermoelectric power $S_{x y}$ and the saturation field. (c) Transverse voltage at $293 \mathrm{~K}$, for different angles $\Theta$. (d) Angular dependence of the saturation transverse voltage $\left(V_{x y}\right.$ at $\left.H_{\text {sat }}\right)$, normalized by its anisotropic response, according to Eq. (1), at different temperatures. The dashed line represents the $\cos \Theta \sin \Theta$ characteristic of PNE, multiplied by a temperature-independent fitting factor. A constant thermoelectric offset voltage was subtracted in (a) and (c) to center the curves at zero voltage when $H=0$.

Moreover, the angular dependence of the PHE shown in Fig. 3(c) is similar to its thermal counterpart (PNE), confirming their common physical origin.

In addition, the maximum AMR should be shifted by $45^{\circ}$ with respect to the PHE, according to [23]

$$
R_{x x}=\rho_{\perp}+\left(\rho_{\|}-\rho_{\perp}\right) \cos ^{2} \Theta .
$$
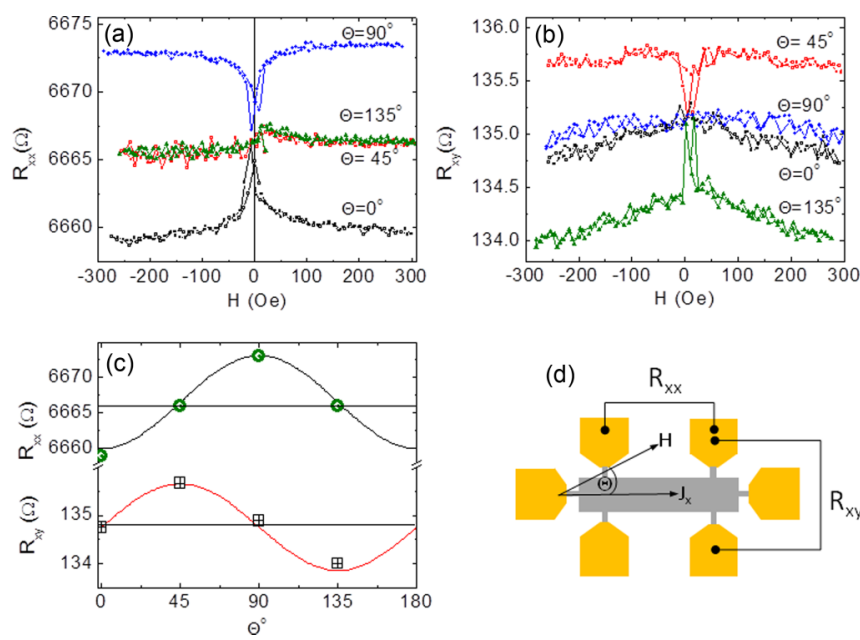

FIG. 3. (Color online) (a) Longitudinal $\left(R_{x x}, \quad \mathrm{AMR}\right)$ and (b) transverse $\left(R_{x y}\right.$, PHE) components of the magnetoresistance at $293 \mathrm{~K}$, along with their angular dependence (c). The sketch in (d) shows the relative orientation of the field and current during the experiment. The dimensions of the film channel in the Hall bar are $100 \times 500 \mu \mathrm{m}^{2}$. The long axis of the Hall bar is along the (110) direction of LSMO film. 

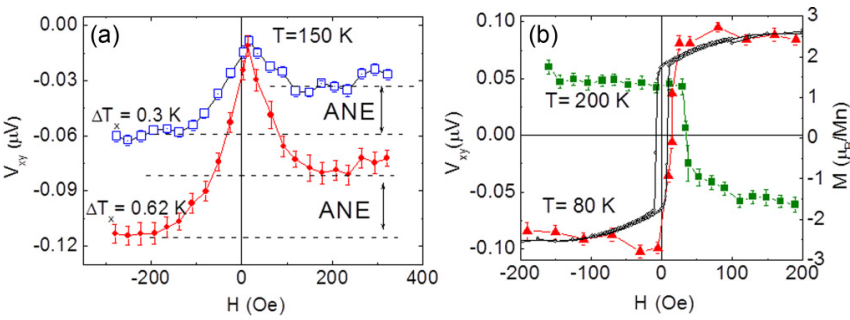

FIG. 4. (Color online) (a) Mixed contribution of the odd ANE and even PNE field dependence, at $150 \mathrm{~K}$, for different in-plane thermal gradients, and $\Delta T_{z} \neq 0$. (b) ANE at $200 \mathrm{~K}$ (squares) and $80 \mathrm{~K}$ (triangles), measured with $\Delta T_{x}=0$ and $\Delta T_{z} \neq 0$. The solid line corresponds to the field dependence of the magnetization at $80 \mathrm{~K}$, with the magnetic field applied along the film plane.

This was also verified experimentally in our LSMO film samples, as shown in Fig. 3(c).

We have also observed a nonsaturating negative MR, at least up to $1.2 \mathrm{~T}$, the maximum field probed in this work, which is already visible in Fig. 3(b). This is commonly observed in thin films of $3 d$ metals, due to the damping of spin waves at high fields, which leads to the reduction of electron-magnon scattering [24]. The same phenomenon was observed in the thermal PNE (see the Supplemental Material, Fig. S4 [21]).

Importantly, we have noticed that even a very small fluctuation $(<0.01 \mathrm{~K})$ in the base temperature of the cryostat is enough to produce a small thermal gradient across the plane of the film $\left(\Delta T_{z} \neq 0\right)$, which introduces an asymmetric response of the transverse voltage with respect to the magnetic field [Fig. 4(a)]. This asymmetric signal shows, in addition to the contribution from the symmetric PNE, an odd function of the magnetic field.

As we mentioned before, in a conducing $\mathrm{FM}$, any $\Delta T_{z} \neq 0$ will create a measurable $V_{x y}$ response due to ANE [Eq. (2)]. This signal should change sign with the magnetic field, and therefore should be responsible for the asymmetric component observed in Fig. 4.

In order to quantify the ANE response in LSMO, a small temperature gradient was intentionally applied perpendicular to the film surface $\left(\Delta T_{z} \neq 0\right)$. The temperature of the $\mathrm{Cu}$ block under the film was varied by less than $1 \mathrm{~K}$, while keeping $\Delta T_{x}=0$. Given the thickness of the film $(\sim 35 \mathrm{~nm})$ it is difficult to estimate an accurate value of the crossplane temperature difference. As shown in Fig. 4(b), a large transverse voltage is observed, in spite of the small temperature difference. The signal is an odd function of the magnetic field, following perfectly the magnetization of the sample, as expected for the ANE.

The temperature difference across the film can now be estimated from the experimental value of $V_{x y}$ and Eq. (2). At $200 \mathrm{~K}$, the Seebeck coefficient of LSMO, $S_{x x}=2.3 \mu \mathrm{V} / \mathrm{K}$ (see the Supplemental Material, Fig. S6 [21]). Using $\xi=$ 0.13 , as for permalloy [12], we obtain $\Delta T_{z} \approx 2 \mu \mathrm{K}$, which corresponds to a gradient of $0.6 \mathrm{~K} / \mathrm{cm}$ across our $35 \mathrm{~nm}$ thick film of LSMO. Note that a substantial error in the estimation of $\xi$ will not change the conclusion, i.e., the extreme sensitivity of the transverse voltage to any parasitic cross-plane temperature gradient. This is an important result, because it demonstrates that very small parasitic thermal currents in magnetic con- ductors may produce thermoelectric voltages in the presence of a transverse magnetic field, which could be relevant in nanodevices.

Moreover, this transverse signal shows the same field and same angular dependence as the SSE, and therefore it must be carefully addressed in conducting ferromagnets. On the other hand, the analysis of the intrinsic magnetothermoelectric response in magnetic insulators can be complicated by the existence of magnetic moments induced at Pt by proximity effects close to the FM/Pt interface $[25,26]$. These results make it very difficult to separate the contribution from ANE and SSE, even in magnetic insulators, and independently of whether a transverse or longitudinal configuration is used to detect the SSE signal $[27,28]$. We repeated the experiments using an Au strip instead of Pt. Au shows a much smaller spinorbit coupling than Pt and therefore will help in discriminating between the intrinsic ANE and SSE signals in LSMO. In our case we have obtained absolutely comparable results using either Pt or Au (see the Supplemental Material, Fig. S5 [21]). Therefore, with our resolution limit we could not detect a signal compatible with SSE in LSMO.

Most important is our observation of a sign change of the ANE with temperature between 200 and $80 \mathrm{~K}$, even though the direction of the thermal gradient and magnetization is kept constant. In fact, comparing Figs. 4(a) and 4(b), it can be seen that the ANE already changes sign between 150 and $200 \mathrm{~K}$. This sign reversal of the ANE was observed in other systems, as semiconducting $\mathrm{Ga}_{1-x} \mathrm{Mn}_{x}$ As [29] and Pt/YIG bilayers [25], and can be understood in terms of the Mott relationship between the anomalous Nernst and Hall coefficients [30-32]. This means that the ANE must be determined by the energy dependence of its electrical charge counterpart:

$$
S_{x y}=\frac{-\pi^{2} k_{B}^{2} T}{3 e}\left(\frac{\partial \ln \rho_{x y}}{\partial E}\right)_{E_{F}},
$$

where $\rho_{x y}=\lambda M_{z} \rho_{x x}^{n}$ is the anomalous Hall resistivity [33,34]. Given the relationship between the anomalous Hall conductivity and the linear resistivity, $\sigma_{x y} \propto \rho_{x x}^{n-2}, n=1$ results in a linear dependence of the Hall conductivity with the scattering time $\tau$, characteristic of the skew scattering extrinsic mechanism for the anomalous Hall effect (AHE) in the clean (high conductivity) limit. When $\tau$ decreases, a transition towards the intrinsic regime $\left(n=2, \sigma_{x y}\right.$ independent of $\tau)$ and the dirty regime $(n=0.4$, hopping conductivity) occurs $[30,35,36]$. Transition metal oxides with intermediate resistivities $\left(\rho_{x x}\right.$ larger than $\left.\approx 10 \mu \Omega \mathrm{cm}\right)$ are expected to fall in the intrinsic regime $(n=2)$ [31].

In the LSMO film, with an in-plane magnetization $M_{x}$ and a perpendicular temperature gradient $\Delta T_{z} \neq 0$, the anomalous Nernst signal of Eq. (2) can be expressed as a function of the other transport coefficients, and assuming $\sigma_{x y}=-\sigma_{y x}$, then [29]

$$
S_{x y}=\frac{\rho_{x y}}{\rho_{x x}}\left(\frac{-\pi^{2} k_{B}^{2} T}{3 e} \frac{\lambda}{\lambda^{\prime}}-(n-1) S_{x x}\right),
$$

where $\lambda^{\prime}=(\partial \lambda / \partial E)$.

Given that the transverse and linear resistivities do not change sign between 150 and $200 \mathrm{~K}$ [34], we can infer 
from the results in Fig. 4 that $n \neq 1$. This is in agreement with previous observations in similar oxides with comparable conductivities, and places LSMO in the intrinsic regime, as expected.

In summary, we have investigated the intrinsic magnetothermoelectric coefficients of half-metallic ferromagnet LSMO thin films and demonstrated experimentally the relationship between the different components of the transport tensors in the presence of a magnetic field. Through a careful control of the thermal gradients in different directions of the samples we have disentangled the contributions of the PNE and ANE to the magnetothermal response of LSMO. Using an $\mathrm{Au}$ metal strip we are able to discard any contribution from the
SSE to our measurements in this technologically important material.

The extreme sensitivity of ANE to $\nabla T_{z}$ could be useful to sense very small temperature differences across nanostructures, which are extremely difficult to measure accurately by other methods. Finally, our results also show the utility of ANE to study the mechanism of the anomalous Hall effect in ferromagnetic metals.

This research was supported by the European Research Council (ERC StrG-259082, 2DTHERMS) and Xunta de Galicia (2012-CP071). We would like to thank M. Abd-Elmeguid, University of Cologne, Germany, for fruitful discussions.
[1] K. Uchida, S. Takahashi, K. Harii, J. Ieda, W. Koshibae, K. Ando, S. Maekawa, and E. Saitoh, Nature (London) 455, 778 (2008).

[2] G. E. Bauer, E. Saitoh, and B. J. van Wees, Nat. Mater. 11, 391 (2012).

[3] K. Uchida, T. Ota, H. Adachi, J. Xiao, T. Nonaka, Y. Kajiwara, G. E. W. Bauer, S. Maekawa, and E. Saitoh, J. Appl. Phys. 111, 103903 (2012).

[4] E. Saitoh, M. Ueda, H. Miyajima, and G. Tatara, Appl. Phys. Lett. 88, 182509 (2006).

[5] L. Gravier, A. Fabian, A. Rudolf, A. Cachin, J.-E. Wegrone, and J.-Ph. Ansernet, J. Magn. Magn. Mater. 271, 153 (2004).

[6] J. M. Ziman, Electrons and Phonons (Oxford University Press, Oxford, U.K., 1960).

[7] K. Uchida, H. Adachi, T. An, T. Ota, M. Toda, B. Hillebrands, S. Maekawa, and E. Saitoh, Nat. Mater. 10, 737 (2011).

[8] C. M. Jaworski, J. Yang, S. Mack, D. D. Awschalom, R. C. Myers, and J. P. Heremans, Phys. Rev. Lett. 106, 186601 (2011).

[9] M. Schmid, S. Srichandan, D. Meier, T. Kuschel, J.-M. Schmalhorst, M. Vogel, G. Reiss, C. Strunk, and C. H. Back, Phys. Rev. Lett. 111, 187201 (2013).

[10] D. Meier, D. Reinhardt, M. Schmid, C. H. Back, J.-M. Schmalhorst, T. Kuschel, and G. Reiss, Phys. Rev. B 88, 184425 (2013).

[11] S. Y. Huang, W. G. Wang, S. F. Lee, J. Kwo, and C. L. Chien, Phys. Rev. Lett. 107, 216604 (2011).

[12] A. Slachter, F. L. Bakker, and B. J. van Wees, Phys. Rev. B 84, 020412(R) (2011).

[13] W. Lin, M. Hehn, L. Chaput, B. Negulescu, S. Andrieu, F. Montaigne, and S. Mangin, Nat. Commun. 3, 744 (2012).

[14] K.-R. Jeon, B.-C. Min, S.-Y. Park, K.-D. Lee, H.-S. Song, Y.-H. Park, and S.-C. Shin, Appl. Phys. Lett. 103, 142401 (2013).

[15] S. Hu and T. Kimura, Phys. Rev. B 87, 014424 (2013).

[16] M. Erehinsky, F. Casanova, I. K. Schuller, and A. Sharoni, Appl. Phys. Lett. 100, 212401 (2012).

[17] J.-H. Park, E. Vescovo, H.-J. Kim, C. Kwon, R. Ramesh, and T. Venkatesan, Nature (London) 392, 794 (1998).

[18] M. Bowen, M. Bibes, A. Barthelemy, J.-P. Contour, A. Anane, Y. Lemaitre, and A. Fert, Appl. Phys. Lett. 82, 233 (2003).

[19] J.-E. Wegrowe, H.-J. Drouhin, and D. Lacour, Phys. Rev. B 89, 094409 (2014).
[20] V. D. Ky, Phys. Status Solidi 22, 729 (1967).

[21] See Supplemental Material at http://link.aps.org/supplemental/ 10.1103/PhysRevB.90.100403 for details on sample preparation and experimental configuration for the measurements.

[22] Y. Suzuki, H. Y. Hwang, S.-W. Cheong, and R. B. van Doner, Appl. Phys. Lett. 71, 140 (1997).

[23] Y. Bason, L. Klein, J.-B. Yau, X. Hong, and C. H. Ahn, Appl. Phys. Lett. 84, 2593 (2004).

[24] B. Raquet, M. Viret, E. Sondergard, O. Cespedes, and R. Mamy, Phys. Rev. B 66, 024433 (2002).

[25] S. Y. Huang, X. Fan, D. Qu, Y. P. Chen, W. G. Wang, J. Wu, T. Y. Chen, J. Q. Xiao, and C. L. Chien, Phys. Rev. Lett. 109, 107204 (2012).

[26] F. Wilhelm, P. Poulopoulos, G. Ceballos, H. Wende, K. Baberschke, P. Srivastava, D. Benea, H. Ebert, M. Angelakeris, N. F. Flevaris, D. Niarchos, A. Rogalev, and N. B. Brookes, Phys. Rev. Lett. 85, 413 (2000)

[27] M. Schreier, N. Roschewsky, E. Dobler, S. Meyer, H. Huebl, R. Gross, and S. T. B. Goennenwein, Appl. Phys. Lett. 103, 242404 (2013).

[28] T. Kikkawa, K. Uchida, Y. Shiomi, Z. Qiu, D. Hou, D. Tian, H. Nakayama, X.-F. Jin, and E. Saitoh, Phys. Rev. Lett. 110, 067207 (2013).

[29] Y. Pu, D. Chiba, F. Matsukura, H. Ohno, and J. Shi, Phys. Rev. Lett. 101, 117208 (2008).

[30] N. F. Mott and H. Jones, The Theory of the Properties of Metals and Alloys (Dover, New York, 1958).

[31] T. Miyasato, N. Abe, T. Fujii, A. Asamitsu, S. Onoda, Y. Onose, N. Nagaosa, and Y. Tokura, Phys. Rev. Lett. 99, 086602 (2007).

[32] Y. Pu, E. Johnston-Halperin, D. D. Awschalom, and J. Shi, Phys. Rev. Lett. 97, 036601 (2006).

[33] P. Matl, N. P. Ong, Y. F. Yan, Y. Q. Li, D. Studebaker, T. Baum, and G. Doubinina, Phys. Rev. B 57, 10248 (1998).

[34] Y. Lyanda-Geller, S. H. Chun, M. B. Salamon, P. M. Goldbart, P. D. Han, Y. Tomioka, A. Asamitsu, and Y. Tokura, Phys. Rev. B 63, 184426 (2001).

[35] S. Onoda, N. Sugimoto, and N. Nagaosa, Phys. Rev. Lett. 97 126602 (2006)

[36] A. Fernández-Pacheco, J. M. De Teresa, J. Orna, L. Morellon, P. A. Algarabel, J. A. Pardo, and M. R. Ibarra, Phys. Rev. B 77, 100403(R) (2008). 